OPEN ACCESS

Edited by:

Yong-Lai Zhang,

Jilin University, China

Reviewed by:

Huhu Cheng,

Tsinghua University, China

Chuangang $\mathrm{Hu}$,

University of New South Wales,

Australia

*Correspondence:

Zhaoshun Gao

gaozs@mail.iee.ac.cn

Specialty section:

This article was submitted to

Nanoscience,

a section of the journal

Frontiers in Chemistry

Received: 05 September 2019 Accepted: 07 November 2019 Published: 10 December 2019

Citation:

Xue J, Gao Z and Xiao L (2019) The Application of Stimuli-Sensitive Actuators Based on Graphene Materials. Front. Chem. 7:803. doi: $10.3389 /$ fchem.2019.00803

\section{The Application of Stimuli-Sensitive Actuators Based on Graphene Materials}

\author{
Jiangli Xue, Zhaoshun Gao* and Liye Xiao \\ Interdisciplinary Research Center, Institute of Electrical Engineering, Chinese Academy of Science, Beijing, China
}

Graphene-based materials that can spontaneously response to external stimulations have triggered rapidly increasing research interest for developing smart devices due to their excellent electrical, mechanical and thermal properties. The specific behaviors as bending, curling, and swing are benefit for designing and fabricating the smart actuation system. In this minireview, we overview and summarize some of the recent advancements of stimuli-responsive actuators based on graphene materials. The external stimulus usually is as electrical, electrochemical, humid, photonic, and thermal. The advancement and industrialization of graphene preparation technology would push forward the rapid progress of graphene-based actuators and broaden their application including smart sensors, robots, artificial muscles, intelligent switch, and so on.

Keywords: graphene, actuators, stimulation, electrical, electrochemical, humidity, light, thermal

\section{INTRODUCTION}

Artificial actuators are stimuli-sensitive devices that can undergo a reversible change in shape, volume, modulus, or some other mechanical properties in response to various external stimuli, which have attracted tremendous attentions attribute to the wide applications for robots, intelligent switch, smart sensors, memory chips, and prosthetic devices (Kim and Lieber, 1999; Jang et al., 2008; Huang et al., 2012; Xue et al., 2015; Xu et al., 2018b). Usually, the external stimuli can be electrical, optical, thermal, humid, and other forms of stimulation depending on the actuation mechanism (Huang et al., 2012). The traditional materials using for actuators generally have their own limitations. For example, piezoelectric or ferroelectric ceramics show low mechanical flexibility leading to slow response, and conducting polymer materials have suffered from high driving voltages and low energy conversion efficiency. Therefore, developing the advanced materials or the compatible fabrication methods for large displacement and a rapid response have been the topic of research during the past decade. To date, carbon-based nanomaterials have been caused great concern for proposing as appropriate candidates for actuators due to the large deformation either in planar or spatial warping (Baughman et al., 1999; Deng et al., 2017). Recently, Peng group reported the mechanically actuating fibers by carbon nanotube displaying a rapid response for using as the smart window and louvers (He et al., 2015).

Graphene, as another carbon nanomaterial with just one atom layer thick, that features high electron conductivity, excellent mechanical flexibility, huge surface area, superior thermal conductivity and stability, has captured a broad research interest for using as material or structural component in the actuator fabrication (Novoselov et al., 2004; Zan et al., 2011; Cheng et al., 2016; Xu et al., 2018b; Liu Y. Q. et al., 2019). It could be not only served as the electrode in the electrically/electrochemically driven actuators, but also the energy converting component or active 
actuation component in the photothermal systems, as well as in the moisture actuators (Yang et al., 2018). For example, the composite graphene fiber can be elongation or contraction under electrochemical stimulus (Wang Y. H. et al., 2013) or serve as a rotational motor under moisture-driven situations (Cheng et al., 2014). As we all know, great progress has been made in studying of actuation system based on graphene materials in only few years. Therefore, to organize an article on graphene-based actuator for quick understanding the limitation and strengths is highly demanded.

In the minireview, we summarize the recent developments of graphene-based actuation systems and the discuss the application of graphene-based actuators under various external stimuli like electrical, electrochemical, humid, light, and other stimulations (Figure 1).

\section{THE APPLICATION OF ACTUATORS UNDER DIFFERENT STIMULI}

Generally, the graphene-based stimuli-responsive actuators are the unique devices that can convert an external stimulus into automatic action. For the sample of isotropy, it shows planar expansion or contraction (Liu et al., 2012b; Hu et al., 2014; Xue et al., 2015), and for the sample with the construction of asymmetric, it shows spatial warping (Liu et al., 2012a; Wang Y. H. et al., 2013). The deformation of graphene is mainly caused by the stretch or rotation of carbon-carbon bands, and the hydrophilic and hydrophobicity of graphenebased materials is another reason inducing the deformation (Xu et al., 2018b). Recently, a novel asymmetric unimorph actuator based on graphene hydrogel was reported exhibiting synchronous responses for the stimulus of both photonic and humidifying/dehumidifying (Yang et al., 2019). In this section, we will briefly summarize the graphene-based actuator induced by some typical external stimuli like electrical, electrochemical, humid, light, and heat.

\section{ELECTRICALLY STIMULATED ACTUATION}

The electrical-stimulus actuators are the most widely research transferring electrical energy/signal to mechanical energy/change (Zhao et al., 2013). Since the related work using graphene as resonator was firstly reported by McEuen group that showing the highest modulus than any other materials (Sazonova et al., 2004; Bunch et al., 2007), graphene materials based electrical stimulussensitive actuators have attracted a great deal of attention. Zhu et al. fabricated a bimorph actuator based on graphene materials with a large displacement and rapid response under low power consumption (Zhu et al., 2011). Later on, Jang group reported an acoustic actuator using a transparent and flexible graphene film as electrode which shown higher responses over all frequencies than those using a commercial PEDOT:PPS film as electrode (Shin et al., 2011). It could be used as loudspeaker with the properties of thin and lightweight.

By using the pure graphene with isotropy, Chen group reported a spongy graphene paper with an inside foldable corrugated structure exhibited reversible macroscopical length contraction with a strain of $2.4 \%$ under $10 \mathrm{~V}$ (Hu et al., 2014). Although the deformation efficiency is large, the power consumption is too much. Soon afterwards, our group put forward a strategy for the controllable fabrication of re-shaped graphene hydrogel showing the large strain up to $2.9 \%$ under very low voltage of $0.8 \mathrm{~V}$ (Xue et al., 2015).

A very recent and interesting study was reported by Wang group (Chang et al., 2019). The soft actuator with the construction of asymmetric based on graphene/polypropylene was fabricated by adhesion the polypropylene film on one side of the drop-coating graphene film. This actuator exhibited large, fast, and reversible deformation in response to electrical stimuli due to their inherent quality for high electrothermal and thermal expansion ability. Furthermore, the biomimetic devices like artificial flowers could be construct by using this actuator, which opens the way for constructing smart devices in the practical biomimetic applications.

\section{ELECTROCHEMICALLY STIMULATED ACTUATION}

Electrochemically stimulated actuator is another widely researched device involving the actuation caused by iondoping. Unimorph actuator with a single layer architecture have been demonstrated previously (Sansiñena et al., 2003; Han and Shi, 2006; Baker et al., 2008). Xie et al. designed and prepared a unique unimorph actuator based on a monolithic graphene film with asymmetrically modified surfaces by treating with $\mathrm{O}_{2}$ and hexane plasma on different sides of graphene film (Xie et al., 2010). Even so, most unimorph actuators consist of a bilayer or multilayer. For example, Liang et al. demonstrated an electrochemical actuator constructed on free-standing graphene $/ \mathrm{Fe}_{3} \mathrm{O}_{4}$ hybrid papers providing increased actuation strain over the pristine graphene papers (Liang et al., 2011).

$\mathrm{Q} u$ group later reported that a kind of actuators made of asymmetrically graphene/polypyrrole films (Liu et al., 2012a) and fibers (Wang Y. H. et al., 2013), which not only exhibiting a large bending angle movement but also for use in multi-armed tweezers. In addition, they also constructed the $3 \mathrm{D}$ uniform network architecture of graphene/polypyrrole actuator with a record strain of $2.5 \%$ (Liu et al., 2012b), even up to $13.5 \%$ (Xue et al., 2015) after re-shaping strategy, under a square wave potential of $0.8 \mathrm{~V}$. These actuators based on the $3 \mathrm{D}$ structure can be used as smart switch and even provide immense potential for the design of new types of actuators.

\section{HUMIDLY STIMULATED ACTUATION}

As we all know, graphene is a kind of hydrophobic material which has been successfully prepared by mechanical exfoliation (Novoselov et al., 2004), chemical vapor deposition on metal (Cu and Ni) substrates (Hao et al., 2013; Lin et al., 2019; Liu C. et al., 2019), epitaxial growth (Yang et al., 2013), and so on. Whereas, graphene oxide as a derivative of graphene is derives from Hummers' method with numerous oxygen-related functional groups inducing fast adsorption and desorption of 


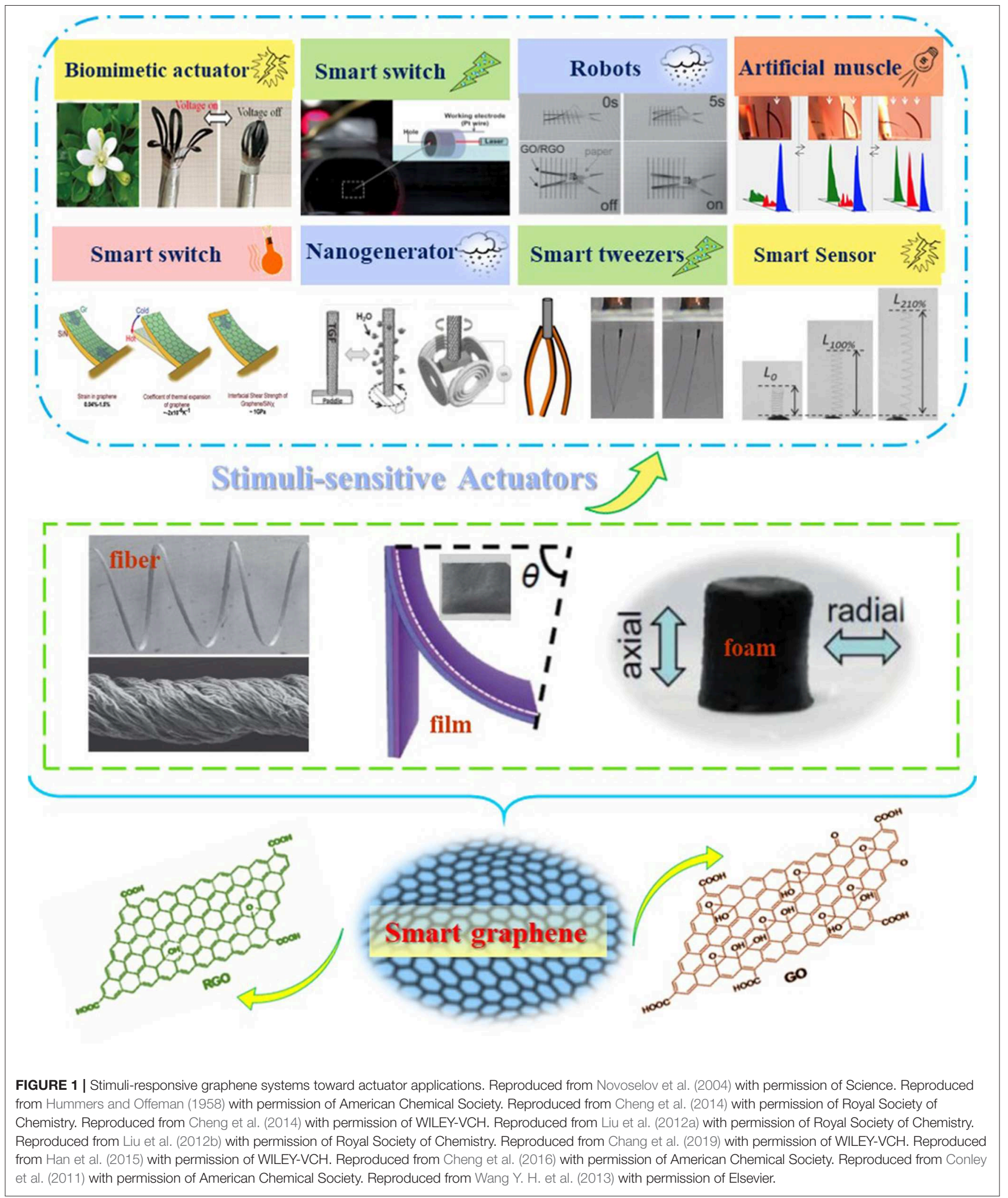

water molecules (Hummers and Offeman, 1958; Zhu et al., 2012; Han et al., 2017). Therefore, another kind of actuators based on the hydrophilic and hydrophobic of graphene materials could be construct. The deformation is mainly related to the change of water amounts or relative humidity in the environment (Zhao et al., 2013). 
Guo et al. investigated GO layers fold and unfold behavior under a humidity changed environment, which opens up a possibility for GO as smart stimuli-response materials (Guo et al., 2011). Later on, Cheng et al. designed a region-asymmetric graphene/graphene oxide fiber actuator via laser reduction of fresh spun graphene oxide fibers (Cheng et al., 2013). By controlling the reduction region-specifically of graphene oxide, the complex deformation of this fiber actuator had been established when the relative humidity changed. Meanwhile, the crawler robot was made which could walk unidirectionally in a step-by-step manner with periodical alternation of the humidity in the environment. In another study, the twist graphene fiber fabricated by rotary of GO fiber can serve as a rotational motor under moisture-driven (Cheng et al., 2014). Before long, the asymmetrical reduced graphene oxide/graphene oxide bilayer structure was constructed by focusing sunlight irradiation on a graphene oxide paper (Han et al., 2015). The similar crawler robot but with the "leg" and "body" was also established that can move forward when the relative humidity change alternatively. Therefore, the moisture-sensitive actuators based on graphene/graphene oxide materials are the potential for developing the application of soft robot.

In recent years, another kind of moisture-sensitive devices maybe called power nanogenerators have attracted much attention through the mechanism is still debatable (Xu et al., 2018b). Qu group reported a lot of related and nice works (Zhao et al., 2015, 2016a, 2017; Liang et al., 2017, 2018; Xu et al., 2018a). For example, Zhao et al. provided electric generators for the first time by using the graphene oxide film with the oxygen-group gradient to harvest energy from moisture environment (Zhao et al., 2015).

\section{LIGHT STIMULI ACTUATION}

Graphene materials could convert the energy of light to heat or mechanical energy and display excellent photo-responsive (Acik et al., 2010; Robinson et al., 2011). An optically driven actuators based on graphene materials can be used as a device with possibilities of wireless actuation, remote displacement controls and robotic motions (Zhao et al., 2013). Wang et al. created near-infrared light-driven actuators based on reduced graphene sheet exhibiting rapid and tunable bending motions at the specific position controlled by light inducing finger-like flexing and crawling (Wang E. et al., 2013). More recent, Liu group designed a bilayer actuator combining the two materials of graphene and PDMS showing the highest deformation. This device was benefit to construct the beluga whale soft robot through improving the photo-responsive deflection and deflecting the speed of these actuators (Wang et al., 2019). From the above, the photo-sensitive actuators based on graphene materials have high deformation and controllable site causing a wide variety application including artificial muscles and soft robots.

\section{THERMALLY STIMULATED ACTUATION}

Theoretically, graphene has demonstrated distinctively large negative coefficient of thermal expansion (CTE). The value is $-7 \mathrm{ppm} \mathrm{K}^{-1}$ in a single-layer (Bao et al., 2009) and $-81.2 \mathrm{ppm}$ $\mathrm{K}^{-1}$ in a discotic liquid crystal phase (Grigoriadis et al., 2010; Zhao et al., 2016b). Conley et al. developed a bilayer actuator constructed by graphene materials attached on different metal substrate like $\mathrm{SiN}_{\mathrm{x}}$ and $\mathrm{Au}$ in order to probe the interaction between graphene and other metal. It was shown that all the cantilevers were bent toward the graphene side at room temperature and the bending degree related to the gradient of temperature, yet the strain was larger based on graphene/SiNx than graphene/Au (Conley et al., 2011). Kim et al. demonstrated a simple method to fabricate graphene oxide/Nylon-6,6 polymer composite fibers which could lift loads over 100 times heavier than itself and exhibit multi-directional actuation with the change of temperature (Kim et al., 2018). Therefore, the novel switch and actuator would be created by using graphene materials under thermal stimuli.

\section{CONCLUSION AND OUTLOOK}

In summary, the recent researches on graphene materials for the devices of actuators have brought up a great deal of government and social attention and support. Particularly, stimuli-sensitive actuators based on graphene materials which spontaneously respond to external stimuli will be given rise to a promising field for developing novel smart devices. The great progress has been made for stimuli-responsive actuation systems that could convert electrical, chemical, photonic, thermal and other types energy/signal to mechanical energy/action. Yet, there are several challenges to overcome like the problem of the graphene production, the unsatisfactory mechanical strength of graphene and the effective of actuation response and so on. Therefore, development of advanced technique and promotion of industrialization are demanded to realize the variety practical applications.

\section{AUTHOR CONTRIBUTIONS}

All authors listed have made a substantial, direct and intellectual contribution to the work, and approved it for publication.

\section{FUNDING}

The authors acknowledge the financial support from National Natural Science Foundation of China under Grant No. 51802303, Dalian National Laboratory for clean Energy (DNL) Cooperation Fund, and Chinese Academy of Science (CAS) under Grant No. DNL 180304. We also acknowledge the financial Hundred-Talent Program (CAS). 


\section{REFERENCES}

Acik, M., Lee, G., Mattevi, C., Chhowalla, M., Cho, K., and Chabal, Y. J. (2010). Unusual infrared-absorption mechanism in thermally reduced graphene oxide. Nat. Mater. 9, 840-845. doi: 10.1038/nmat2858

Baker, C. O., Shedd, B., Innis, P. C., Whitten, P. G., Spinks, G. M., Wallace, G. G., et al. (2008). Monolithic actuators from flash-welded polyaniline nanofibers. Adv. Mater. 20, 155-158. doi: 10.1002/adma.200602864

Bao, W., Miao, F., Chen, Z., Zhang, H., Jang, W., Dames, C., et al. (2009). Controlled ripple texturing of suspended graphene and ultrathin graphite membranes. Nat. Nanotechnol. 4, 562-566. doi: 10.1038/nnano.2009.191

Baughman, R. H., Cui, C., Zakhidov, A. A., Iqbal, Z., Barisci, J. N., Spinks, G. M., et al. (1999). Carbon nanotube actuators. Science 284, 1340-1344. doi: $10.1126 /$ science.284.5418.1340

Bunch, J. S., van der Zande, A. M., Verbridge, S. S., Frank, I. W., Tanenbaum, D. M., Parpia, J. M., et al. (2007). Electromechanical resonators from graphene sheets. Science 315, 490-493. doi: 10.1126/science.1136836

Chang, L., Huang, M., Qi, K., Jing, Z., Yang, L., Lu, P., et al. (2019). Graphene-based bimorph actuators with dual-response and large-deformation by a simple method. Macromol. Mater. Eng. 304:1800688. doi: 10.1002/mame.201800688

Cheng, H. H., Hu, Y., Zhao, F., Dong, Z. L., Wang, Y. H., Chen, N., et al. (2014). Moisture-activated torsional graphene-fiber motor. Adv. Mater. 26, 2909-2913. doi: 10.1002/adma.201305708

Cheng, H. H., Liu, J., Zhao, Y., Hu, C. G., Zhang, Z. P., Chen, N., et al. (2013). Graphene fibers with predetermined deformation as moisturetriggered actuators and robots. Angew. Chem. Int. Ed. Engl. 52, 10482-10486. doi: $10.1002 /$ anie. 201304358

Cheng, H. H., Zhao, F., Xue, J. L., Shi, G., Jiang, L., and Qu, L. T. (2016). One single graphene oxide film for responsive actuation. ACS Nano 10, 9529-9535. doi: 10.1021/acsnano.6b04769

Conley, H., Lavrik, N. V., Prasai, D., and Bolotin, K. I. (2011). Graphene bimetallic-like cantilevers: probing graphene/substrate interactions. Nano Lett. 11, 4748-4752. doi: $10.1021 / \mathrm{nl} 202562 \mathrm{u}$

Deng, J., Xu, Y., He, S., Chen, P., Bao, L., Hu, Y., et al. (2017). Preparation of biomimetic hierarchically helical fiber actuators from carbon nanotubes. Nat. Protoc. 12, 1349-1358. doi: 10.1038/nprot.2017.038

Grigoriadis, C., Haase, N., Butt, H. J., Mullen, K., and Floudas, G. (2010). Negative thermal expansion in discotic liquid crystals of nanographenes. Adv. Mater. 22, 1403-1406. doi: 10.1002/adma.200903264

Guo, F., Kim, F., Han, T. H., Shenoy, V. B., Huang, J., and Hurt, R. H. (2011). Hydration-responsive folding and unfolding in graphene oxide liquid crystal phases. ACS Nano 5, 8019-8025. doi: 10.1021/nn2025644

Han, D. D., Zhang, Y. L., Jiang, H. B., Xia, H., Feng, J., Chen, Q. D., et al. (2015). Moisture-responsive graphene paper prepared by self-controlled photoreduction. Adv. Mater. 27, 332-338. doi: 10.1002/adma.201403587

Han, G. Y., and Shi, G. Q. (2006). Electrochemical actuator based on single-layer polypyrrole film. Sensors Actuat. B 113, 259-264. doi: 10.1016/j.snb.2005.02.055

Han, Q., Chen, N., Zhang, J., and Qu, L. T. (2017). Graphene/graphitic carbon nitride hybrids for catalysis. Mater. Horizons 4, 832-850. doi: 10.1039/C7MH00379J

Hao, Y., Bharathi, M. S., Wang, L., Liu, Y., Chen, H., Nie, S., et al. (2013). The role of surface oxygen in the growth of large single-crystal graphene on copper. Science 342, 720-723. doi: 10.1126/science.1243879

He, S. S., Chen, P. N., Qiu, L. B., Wang, B. J., Sun, X. M., Xu, Y. F., et al. (2015). A mechanically actuating carbon-nanotube fiber in response to water and moisture. Angew. Chem. Int. Edn. 54, 14880-14884. doi: $10.1002 /$ anie. 201507108

Hu, Y., Lan, T., Wu, G., Zhu, Z., Tao, X., and Chen, W. (2014). Novel electromechanical actuation based on a spongy graphene paper. Chem. Commun. 50, 4951-4954. doi: 10.1039/c3cc49376h

Huang, Y., Liang, J. J., and Chen, Y. S. (2012). The application of graphene based materials for actuators. J. Mater. Chem. 22, 3671-3679. doi: $10.1039 / \mathrm{c} 2 \mathrm{jm} 15536 \mathrm{~b}$

Hummers, W. S., and Offeman, R. E. (1958). Preparation of graphitic oxide. J. Am. Chem. Soc. 80, 1339-1339. doi: 10.1021/ja01539a017

Jang, J. E., Cha, S. N., Choi, Y. J., Kang, D. J., Butler, T. P., Hasko, D. G., et al. (2008). Nanoscale memory cell based on a nanoelectromechanical switched capacitor. Nat. Nanotechnol. 3, 26-30. doi: 10.1038/nnano.2007.417
Kim, H., Moon, J. H., Mun, T. J., Park, T. G., Spinks, G. M., Wallace, G. G., et al. (2018). Thermally responsive torsional and tensile fiber actuator based on graphene oxide. ACS Appl. Mater. Interfaces 10, 32760-32764. doi: 10.1021 /acsami.8b12426

Kim, P., and Lieber, C. M. (1999). Nanotube nanotweezers. Science 286, 2148-2150. doi: $10.1126 /$ science.286.5447.2148

Liang, J. J., Huang, Y., Oh, J. Y., Kozlov, M., Sui, D., Fang, S. L., et al. (2011). Electromechanical actuators based on graphene and graphene/Fe3O4 hybrid paper. Adv. Funct. Mater. 21, 3778-3784. doi: 10.1002/adfm.201101072

Liang, Y., Zhao, F., Cheng, Z., Deng, Y., Xiao, Y., Cheng, H., et al. (2018). Electric power generation via asymmetric moisturizing of graphene oxide for flexible, printable and portable electronics. Energy Environ. Sci. 11, 1730-1735. doi: 10.1039/C8EE00671G

Liang, Y., Zhao, F., Cheng, Z., Zhou, Q., Shao, H., Jiang, L., et al. (2017). Selfpowered wearable graphene fiber for information expression. Nano Energy 32, 329-335. doi: 10.1016/j.nanoen.2016.12.062

Lin, L., Zhang, J., Su, H., Li, J., Sun, L., Wang, Z., et al. (2019). Towards super-clean graphene. Nat. Commun. 10:1912. doi: 10.1038/s41467-019-09565-4

Liu, C., Xu, X., Qiu, L., Wu, M., Qiao, R., Wang, L., et al. (2019). Kinetic modulation of graphene growth by fluorine through spatially confined decomposition of metal fluorides. Nat. Chem. 11, 730-736. doi: 10.1038/s41557-019-0290-1

Liu, J., Wang, Z., Xie, X. J., Cheng, H. H., Zhao, Y., and Qu, L. T. (2012a). A rationally-designed synergetic polypyrrole/graphene bilayer actuator. J. Mater. Chem. 22, 4015-4020. doi: 10.1039/c2jm15266e

Liu, J., Wang, Z., Zhao, Y., Cheng, H., Hu, C., Jiang, L., et al. (2012b). Threedimensional graphene-polypyrrole hybrid electrochemical actuator. Nanoscale 4, 7563-7568. doi: 10.1039/c2nr32699j

Liu, Y. Q., Chen, Z. D., Mao, J. W., Han, D. D., and Sun, X. (2019). Laser fabrication of graphene-based electronic skin. Front. Chem. 7:461 doi: $10.3389 /$ fchem. 2019.00461

Novoselov, K. S., Geim, A. K., Morozov, S. V., Jiang, D., Zhang, Y., Dubonos, S. V., et al. (2004). Electric field effect in atomically thin carbon films. Science 306, 666-669. doi: 10.1126/science.1102896

Robinson, J. T., Tabakman, S. M., Liang, Y., Wang, H., Casalongue, H. S., Vinh, D., et al. (2011). Ultrasmall reduced graphene oxide with high near-infrared absorbance for photothermal therapy. J. Am. Chem. Soc. 133, 6825-6831. doi: $10.1021 /$ ja2010175

Sansiñena, J. M., Gao, J., and Wang, H. L. (2003). High-performance, monolithic polyaniline electrochemical actuators. Adv. Funct. Mater. 13, 703-709. doi: $10.1002 /$ adfm.200304347

Sazonova, V., Yaish, Y., Üstünel, H., Roundy, D., Arias, T. A., and McEuen, P. L. (2004). A tunable carbon nanotube electromechanical oscillator. Nature 431, 284-287. doi: 10.1038/nature02905

Shin, K. Y., Hong, J. Y., and Jang, J. (2011). Flexible and transparent graphene films as acoustic actuator electrodes using inkjet printing. Chem. Commun. 47, 8527-8529. doi: 10.1039/c1cc12913a

Wang, E., Desai, M. S., and Lee, S. W. (2013). Light-controlled grapheneelastin composite hydrogel actuators. Nano Lett. 13, 2826-2830. doi: $10.1021 / \mathrm{nl} 401088 \mathrm{~b}$

Wang, X., Jiao, N., Tung, S., and Liu, L. (2019). Photoresponsive graphene composite bilayer actuator for soft robots. ACS Appl. Mater. Interfaces. 11:33. doi: 10.1021/acsami.9b09491

Wang, Y. H., Bian, K., Hu, C. G., Zhang, Z. P., Chen, N., Zhang, H. M., et al. (2013). Flexible and wearable graphene/polypyrrole fibers towards multifunctional actuator applications. Electrochem. Commun. 35, 49-52. doi: 10.1016/j.elecom.2013.07.044

Xie, X. J., Qu, L. T., Zhou, C., Li, Y., Zhu, J., Bai, H., et al. (2010). An asymmetrically surface-modified graphene film electrochemical actuator. ACS Nano 4, 6050-6054. doi: 10.1021/nn101563x

Xu, T., Ding, X., Shao, C., Song, L., Lin, T., Gao, X., et al. (2018a). Electric power generation through the direct interaction of pristine grapheneoxide with water molecules. Small 14:e1704473. doi: 10.1002/smll.201 704473

Xu, T., Han, Q., Cheng, Z. H., Zhang, J., and Qu, L. T. (2018b). Interactions between graphene-based materials and water molecules toward actuator and electricity-generator applications. Small Methods 2:1800108. doi: $10.1002 /$ smtd.201800108 
Xue, J. L., Hu, C. G., Lv, L. X., Dai, L. M., and Qu, L. T. (2015). Re-shaping graphene hydrogels for effectively enhancing actuation responses. Nanoscale 7, 12372-12378. doi: 10.1039/C5NR02604K

Yang, L. L., Qi, K., Chang, L. F., Xu, A. F., Hu, Y., Zhai, H., et al. (2018). A powerful dual-responsive soft actuator and photo-to-electric generator based on graphene micro-gasbags for bioinspired applications. J. Mater. Chem. B 6, 5031-5038. doi: 10.1039/С8ТB01222A

Yang, N., Ji, X., Sun, J., Zhang, Y., Xu, Q., Fu, Y., et al. (2019). Photonic actuators with predefined shapes. Nanoscale 11, 10088-10096. doi: 10.1039/C9NR02294E

Yang, W., Chen, G., Shi, Z., Liu, C. C., Zhang, L., Xie, G., et al. (2013). Epitaxial growth of single-domain graphene on hexagonal boron nitride. Nat. Mater. 12, 792-797. doi: $10.1038 /$ nmat3695

Zan, R., Bangert, U., Ramasse, Q., and Novoselov, K. S. (2011). Metal-graphene interaction studied via atomic resolution scanning transmission electron microscopy. Nano Lett. 11, 1087-1092. doi: 10.1021/nl103980h

Zhao, F., Cheng, H., Zhang, Z., Jiang, L., and Qu, L. (2015). Direct power generation from a graphene oxide film under moisture. Adv. Mater. 27, 4351-4357. doi: 10.1002/adma.201501867

Zhao, F., Liang, Y., Cheng, H. H., Jiang, L., and Qu, L. T. (2016a). Highly efficient moisture-enabled electricity generation from graphene oxide frameworks. Energy Environ. Sci. 9, 912-916. doi: 10.1039/C5EE03701H

Zhao, F., Wang, L., Zhao, Y., Qu, L., and Dai, L. (2017). Graphene oxide nanoribbon assembly toward moisture-powered information storage. Adv. Mater. 29:1604972. doi: 10.1002/adma.201604972
Zhao, F., Zhao, Y., Chen, N., and Qu, L. T. (2016b). Stimuli-deformable graphene materials: from nanosheet to macroscopic assembly. Mater. Today 19, 146-156. doi: 10.1016/j.mattod.2015.10.010

Zhao, Y., Song, L., Zhang, Z. P., and Qu, L. T. (2013). Stimulus-responsive graphene systems towards actuator applications. Energy Environ. Sci. 6, 3520-3536. doi: 10.1039/c3ee42812e

Zhu, J., Andres, C. M., Xu, J., Ramamoorthy, A., Tsotsis, T., and Kotov, N. A. (2012). Pseudonegative thermal expansion and the state of water in graphene oxide layered assemblies. ACS Nano 6, 8357-8365. doi: 10.1021/nn30 31244

Zhu, S. E., Shabani, R., Rho, J., Kim, Y., Hong, B. H., Ahn, J. H., et al. (2011). Graphene-based bimorph microactuators. Nano Lett. 11, 977-981. doi: $10.1021 / \mathrm{nl} 103618 \mathrm{e}$

Conflict of Interest: The authors declare that the research was conducted in the absence of any commercial or financial relationships that could be construed as a potential conflict of interest.

Copyright $\odot 2019$ Xue, Gao and Xiao. This is an open-access article distributed under the terms of the Creative Commons Attribution License (CC BY). The use, distribution or reproduction in other forums is permitted, provided the original author(s) and the copyright owner(s) are credited and that the original publication in this journal is cited, in accordance with accepted academic practice. No use, distribution or reproduction is permitted which does not comply with these terms. 